\title{
Pulsar searches in nearby dwarf spheroidal galaxies
}

\author{
Eduardo Rubio-Herrera ${ }^{1}$ and Thomas Maccarone ${ }^{2}$ \\ ${ }^{1}$ Instituto de Astronomía, Universidad Nacional Autónoma de México \\ Apartado Postal 70-264, CP04510, México DF, México \\ email: erubio@astro.unam.mx \\ ${ }^{2}$ School of Physics \& Astronomy, University of Southampton, \\ Highfield, Southampton S017 1BJ United Kingdom \\ email: t.j.maccarone@soton.ac.uk
}

\begin{abstract}
We have been undertaking a comprehensive survey for pulsars and fast radio transients in the dwarf spheroidal satellite galaxies of the Milky Way using the Green Bank Radio Telescope operating at a central frequency of $350 \mathrm{MHz}$. Our search pipeline allows the detection of periodical signals and single dispersed pulses and it is optimized to search for millisecond radio pulsars. Here we present preliminary results of the searches we have conducted in the Ursa Minoris, Draco and Leo I dwarf spheroidal satellite galaxies. Our searches have revealed no periodic signals but a few unconfirmed millisecond single pulses at various dispersion measures, possibly related to neutron stars. Detecting neutron stars in these systems can potentially help to test the existence of haloes of dark matter surrounding these systems as predicted by Dehnen \& King (2006).
\end{abstract}

Keywords. galaxies: dwarf spheroidals, -stars: neutron, -pulsars: general, searches, individual pulses.

\section{Introduction}

Searches for any manifestation of neutron stars at radio wavelengths such as pulsars, in nearby galaxies can help us to probe insightful information about the stellar content, dynamics and and about the intergalactic medium surrounding them. Until recently, only a few of the satellites of our Milky Way have been searched for pulsars and these searches have been concentrated in the Magellanic Clouds resulting in the discovery of a few tens of new pulsars (McCulloch et al. 1983, Manchester et al. 2006 and references therein). This high detection rate is mainly because of the large star formation rates of these clouds. Searches for pulsars in galaxies other than the Magellanic Clouds have been hampered by the enormous distances at which these objects lie, and by the limitations in the dwell times of the observations which constrain the $\mathrm{S} / \mathrm{N}$ at which these objects can be detected. Some previous attempts of detecting pulsars in these objects are the observations made in the Fornax dwarf spheroidal by McLaughlin \& Cordes (2003) which resulted in no detections.

Despite the fact that dwarf spheroidal galaxies (hereafter dSph) have had almost no recent episodes of stellar formation and that these objects display low metallicities, we have two main motivations for searching pulsars in these objects. The first one is the discovery of 5 low-mass X-ray binaries (LMXBs), in the Sculptor Spheroidal Dwarf Galaxy (Maccarone et al. 2005) which proves that this kind of object can exist in an old stellar population, an environment where star formation and stellar encounters are low. The fact that the distances and stellar populations of the dwarf companion galaxies have been well established makes these objects ideal places for the search of rare classes 
of binary stars and their descendants such as millisecond pulsars (MSPs). Our second motivation is that finding neutron stars in the dSph galaxies, can potentially help us to investigate the dark matter component of these galaxies as we explain. When neutron stars are formed during the Type II supernova explosion, they get a typically kick velocity that varies between $20-200 \mathrm{~km} \mathrm{~s}^{-1}$ (Podsiadlowski et al. 2005) which exceeds the velocity dispersion of the stellar components in the dSph of $\sim 6 \mathrm{~km} \mathrm{~s}^{-1}$. Dehnen \& King (2006) proved that if these galaxies have haloes of dark matter, there should be also haloes of neutron stars at highly eccentric orbits, composed by either Low Mass X-ray Binaries (LMXBs), MSPs which are direct descendants from LMXBs and perhaps some old and still active radio pulsar. This prediction can be tested implementing a systematic search for any manifestation of pulsars in these galaxies at radio wavelengths.

Many factors may hemper these searches such as the enormous distances at which the dSph are located, the very low numbers of MSPs within them, the beaming effects and low luminosities. However to increase the chance of detecting these pulsars and get an idea of their spatial distribution within these galaxies with the current facilities, one could observe many (if not all) the dSph satellites of our Galaxy. We have initiated a campaign to observe some of these galaxies and here we present our preliminary results.

\section{Observations and data reduction}

A summary of the observations of the dSph galaxies reported here is shown in Table 1. All these observations were made using the Green Bank Radio Telescope (GBT) operating at a central frequency of $350 \mathrm{MHz}$ and using the GUPPI pulsar card. At this frequency the beam size of the GBT is about $0.6^{\circ}$, much larger than the angular size of the main bulge of our targets. In every observation we have sampled the data at $81.92 \mu \mathrm{s}$ using a bandwidth $\Delta f=100 \mathrm{MHz}$ across 4096 frequency channels. Our observations were reduced using the PRESTO package (Ransom et al. 2002) applying periodicity searches optimized for detecting MSPs. We have also implemented single pulse searches in our reduction pipeline. For the reduction we have used the cluster ATOCATL located at the Institute of Astronomy of the National Autonomous University of Mexico.

Our reduction process starts by finding radio frequency interference (RFI) in our data and removing frequencies related to RFI. Later we prepare timeseries for all the trial dispersion measures (DMs) in which we perform the searches. The trial DMs are spaced adequately in order to maximize the sensitivity of the data to millisecond pulsars. We have produced a total of 12468 trial DM timeseries with step sizes of $0.05,0.1$ and $0.3 \mathrm{pc}$ $\mathrm{cm}^{-3}$, each corresponding to the first and second $\mathrm{DM}$ diagonal located at $\mathrm{DM}=346.50$ and $\mathrm{DM}=600 \mathrm{pc} \mathrm{cm}^{-3}$ (the point where the dispersion delay across a frequency channel is equal to twice the sampling time). We now explain the basics of our searching methods. A detailed explanation of our pipeline analysis and its sensitivity will be published elsewhere. For the periodicity searches we have used an FFT algorithm that searches for all the relevant spectral features which are stored and used later to search for accelerations summing up to 8 harnmonics. At this point we search the data in segments of 10, 20 minutes to be senstive to systems with short orbital periods. For systems consisting of weak/distant pulsars we also analyzed the dat using segments of 60 and $120 \mathrm{~min}$.

For the single pulse searches we have used the routine SINGLE PULSE SEARCH of PRESTO. This routine attempts to find single pulses by applying a match filter to the data with a series of boxcar functions of different widths. For our searches we add 1,2,3,4,6,9,14, $20,30,45,70$ and 100 bins of data in order to be sensitive to pulses of different width 
Table 1. GBT observations of the dSph galaxies satellites presented here

\begin{tabular}{lcccrrrc}
\hline Galaxy & $\begin{array}{c}\text { RA (J2000.0) } \\
\text { (h.m.s.) }\end{array}$ & $\begin{array}{c}\text { DEC (J2000.0) } \\
\text { (d.m.s.) }\end{array}$ & $\begin{array}{c}\ell \\
(\mathrm{deg})\end{array}$ & $\begin{array}{c}b \\
(\mathrm{deg})\end{array}$ & $\begin{array}{c}\mathrm{N}_{p} \\
(\mathrm{kpc})\end{array}$ & $\begin{array}{c}\text { Total Dwell } \\
\text { Time }(\mathrm{s})\end{array}$ \\
\hline Ursa Minoris & $15: 09: 08.04$ & $+67: 13: 21.36$ & 104.9662 & 44.8012 & 1 & 60 & 17610 \\
Draco & $17: 20: 12.12$ & $+57: 54: 55.80$ & 86.3681 & 34.7226 & 2 & 80 & 15670 \\
Leo I & $10: 08: 27.00$ & $+12: 18: 27.36$ & 225.9816 & 49.1090 & 5 & 250 & 64000 \\
\hline
\end{tabular}

$(W)$. This routine filters duplicate candidates and records only the most significant ones $(\mathrm{S} / \mathrm{N}>5)$, this threshold is chosen following the statistics for single pulses explained in Cordes \& McLaughlin (2003). We have excised from the timeseries all the times where RFI was identified. All the relevant pulses that match this criteria are recorded and inspected later.

\section{Preliminary results and discussion}

We have completed the periodicity searches and single pulse analysis of all the galaxies listed in Table 1. These observations are part of a larger data set of dwarf spheroidal galaxies that is being reduced now and that will be published elsewhere. Our searches have revealed so far none MSPs or normal pulsars and a few single pulses. Three of the best detections were recorded in are the direction of the Ursa Minoris dSph and are shown in Figure 1. These unconfirmed sources may be of extragalactic origin (i.e. outside our Galaxy and not in the Ursa Minor dSph) or some unknown form of interference. The argument in favour of their extragalactic origin is the high DM they show. Given the large column density required, compared with the observational constraints on the column density in the Ursa Minor galaxy (Gallagher et al. 2003), in the future we plan to take additional care to ensure that these pulses are real astrophysical sources by analyzing the spectrum of the individual single pulses. We show here preliminary results, a more detailed analysis of our results will be presented elswhere.
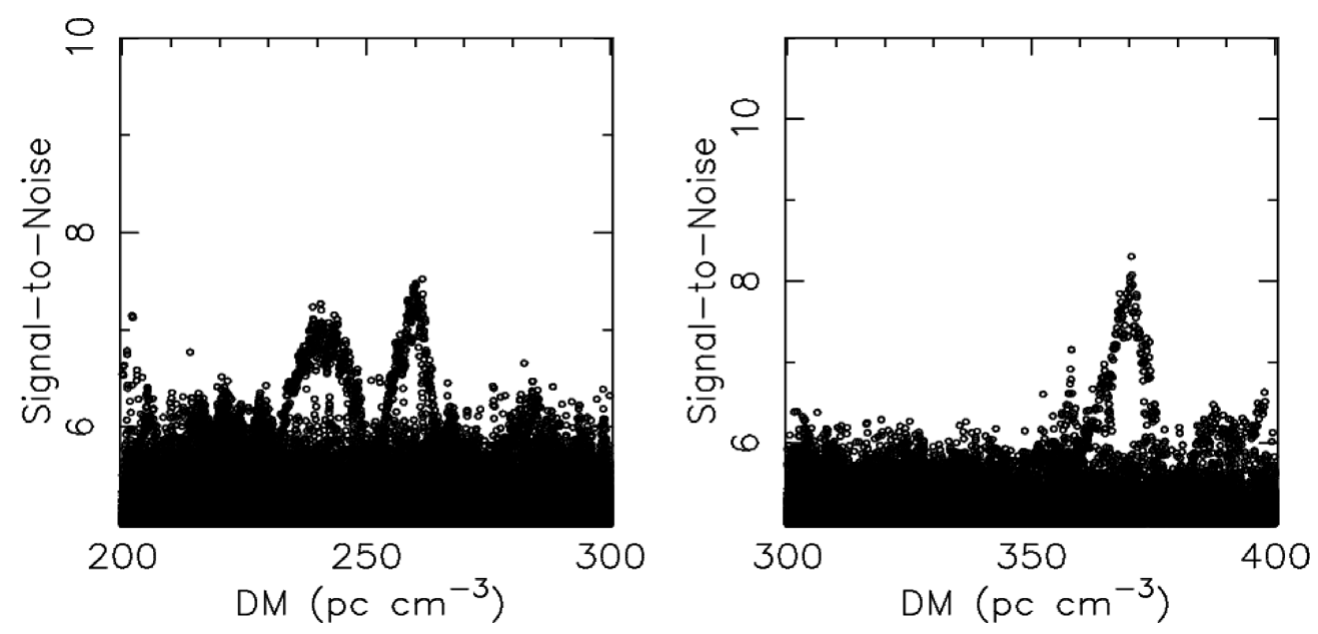

Figure 1. Three examples of bright pulses detected in the Ursa Minoris dSph galaxy. The high $\mathrm{DM}$ of these bursts $\left(\mathrm{DM}>200 \mathrm{pc} \mathrm{cm}^{-3}\right.$ ) locates them well outside our Galaxy. In the left we show two pulses detected with a $\mathrm{S} / \mathrm{N}>7.0$ while in the right one detected with a $\mathrm{S} / \mathrm{N}>8$. These pulses were not detected in other observations. 


\section{Conclusions and future work}

We have presented some preliminary results of a work in progress. We have shown here that searches for pulsars in dSph galaxies can offer us a unique possibility to probe the intergalactic medium (by constraining the electronic content of it) and the stellar content of these galaxies by confirming the existence of MSPs or normal pulsars. If any of these objects is detected and we locate it, we could establish if they form haloes and confirm the predictions made by Dehnen \& King (2006). The fact that we sample the radio sky for long periods also increases our chances of detecting rare and perhaps new transient sources such as the so-called Lorimer burst (Lorimer et al. 2007). For the future we aim to:

- Finish the reduction of more data taken from other dSph that will be collected at the GBT, as well as the reduction of archival data taken with the GBT and the Arecibo radio telescopes of a few other $\mathrm{dSph}$ galaxies.

- Develop synthetic models to describe the pulsar population of the dSph galaxies in order to predict the number of detectable neutron stars in these systems. We aim to be able to establish the probability of successful detections of MSPs and normal pulsars with the current facilities such as the Parkes, GBT and the Arecibo radio telescopes.

- Investigate these objects with larger instruments. The outstanding sensitivity that LOFAR will have (see Stappers et al. 2011), and the proposed Square Kilometre Array (SKA) may confirm the existence of MSPs in these dSph galaxies. With appropriate timing and sufficient observations it may be possible to establish their spatial distribution and perhaps the dynamics of these systems which in turn can be used to constrain the shape and distribution of dark matter in these objects.

\section{Acknowledgements}

The GBT is operated by the National Radio Astronomy Observatory, a facility of the National Science Foundation operated under cooperative agreement by Associate Universities Inc. We thank S. Ransom and the GBT operators for their help in planning and executing these observations. ERH is a DGAPA-UNAM fellow and acknowledges support from IAU GA993 and CONACYT for this conference.

\section{References}

Cordes, J. M. \& McLaughlin, M. A. 2003, ApJ, 596, 1142

Cordes, J. M. \& Lazio, T. J. W. 2002, ArXiv preprint, http://xxx.lanl.gov/abs/astro-ph/0207156 Dehnen, W. \& King, A. 2006, MNRAS, 367, L29

Gallagher, J. S., Madsen, G. J., Reynolds, R. J., Grebel, E. K., \& Smecker-Hane, T. A. 2003, ApJ, 588, 326

Lorimer, D. R., Bailes, M., McLaughlin, M., et al. 2007, Science, 318, 777

Maccarone, T. J., Kundu, A., Zepf, S. E., Piro, A. L., \& Bildsten, L. 2005, MNRAS, 364, L61

Manchester, R. N., Fan, G., Lyne, A., Kaspi, V. M., \& Crawford, F. 2006, ApJ, 649, 235

McCulloch, P. M., Hamilton, P. A., Ables, J. G., \& Hunt, A. J. 1983, Nature, 303, 307

McLaughlin, M. A. \& Cordes, J. M. 2003, ApJ, 596, 982

Podsiadlowski P., Pfahl E., \& Rappaport S. 2005, in Rasio, F. A., Stairs, I. H., eds, ASP Conf. Ser. Vol. 328, Binary Radio pulsars. Astron. Soc. Pac., San Francisco, p.327

Ransom, S. M., Eikenberry, S. S., \& Middleditch, J. 2002, AJ, 124, 1788

Stappers, B. W., Hessels, J. W. T., Alexov, A., et al. 2011, A\&GA, 530, A80 